\title{
Binary Consensus Through Binary Communication
}

\author{
Jemin George and Ananthram Swami
}

\begin{abstract}
The problem of binary consensus for an undirected, synchronous, fixed topology network through noiseless, binary communication is the focus of this paper. We propose a binary consensus protocol that guarantees network convergence to the initial network majority via binary communication among one-hop neighbors. The proposed protocol requires the nodes to keep track of an internal state, which accounts for the local disagreement and the local average of the network vote. Convergence of the algorithm to the initial global majority is proved using the lossy $S$-procedure. Numerical simulations are included to demonstrate the performance of the proposed protocol.
\end{abstract}

\section{INTRODUCTION}

Until now, it was believed that a networked set of agents holding binary opinions is unable to compute the majority opinion by means of local binary interactions. This algorithmic problem is widely known as binary consensus, where, initially, each node in the network holds one of two states and the goal for each node is to correctly decide which one of the two states was initially held by the majority of the nodes. One of the solutions to the binary consensus problem is a quantized gossip algorithm, which is guaranteed to reach interval consensus if the nodes are allowed to communicate a quaternary state instead of a binary state [1]. Analysis of a quantized gossip type algorithm for binary consensus on a complete graph using three states for signaling and memory is presented in [2]. An extension of the binary consensus algorithm presented in [1] for the ternary voting problem using 15 states and the quaternary voting problem using 100 states is given in [3]. This quantized gossip algorithm has been extensively studied for its convergence rate [4], [5] and has been implemented on a real wireless sensor network testbed under the TinyOS environment [6], [7].

A binary consensus based distributed detection scheme for adhoc sensor networks is proposed in [8]. Compared to the quantized gossip algorithm, the binary consensus protocol given in [8] only communicates a binary state but does not guarantee convergence to the majority. Among the different binary consensus protocols, the simplest algorithm comprises of individual nodes calculating the local average and voting one if the local average is greater than or equal to $1 / 2$ and zero otherwise. The performance of such a binary consensus protocol for a fully connected network in the presence of Gaussian communication noise is considered in [9]. Extensions of the binary consensus protocol of [9] to accommodate for noise and link qualities are given in [10]-[12] and the fully connected network assumption is relaxed in [13]. Application of a binary consensus protocol for distributed detection of primary users in cognitive radio networks is presented in [14]. More recently, the problem of distributed hypothesis testing in a cooperative network of agents via binary communication is studied in [15]. Though the algorithm in [15] has been shown to almost surely reach consensus on a binary state, it is known to fail for certain initial states [16] and convergence to a global majority is yet to be proven.

The formulation and analysis of a binary consensus protocol for an undirected, synchronous, fixed topology network through noise-

J. George and A. Swami are with the U.S. Army Research Laboratory, 2800 Powder Mill Rd, Adelphi, MD 20783-1197, USA. \{jemin.george \& ananthram.swami\}.civ@mail.mil

U.S. Government work not protected by U.S. copyright less, binary communication is considered in this paper. Traditionally, average consensus algorithms are based on local disagreement between nodes [17], [18], while the binary consensus protocols are based on the local average among the nodes [9], [13]. The proposed binary consensus protocol involves an internal learning state associated with each node whose values are updated based on both local disagreement and local average of the binary votes. The algorithm presented here contains two design parameters $\alpha$, which indicates how fast an initial binary state is forgotten within a node, and $\gamma$, which decides how fast learning occurs within the node. Convergence analysis of the proposed scheme is conducted using the $S$-procedure and convergence to the global majority is proven.

The structure of this paper is as follows. A brief overview of the binary consensus problem and some preliminary results are given in section II. Formulation of the distributed binary consensus protocol and its performance analysis are presented in section III. Afterwards, numerical simulations are presented in section IV to further illustrate the performance of the proposed algorithm. Finally concluding remarks are given in section $\mathrm{V}$.

\section{Binary Consensus Problem}

The problem of binary consensus involves an undirected network of $n$ nodes with an initial binary voting, which is indicated by an $n$-dimensional binary vector $\mathbf{b}_{0} \in\{0,1\}$. The nodes are said to reach binary consensus at time $k^{*}$, if

$$
\mathbf{b}_{0}^{T} \mathbf{b}_{0}>n / 2 \quad \Rightarrow \quad \mathbf{b}(k)=\mathbf{1}_{n}, \quad \forall k \geq k^{*},
$$

and if

$$
\mathbf{b}_{0}^{T} \mathbf{b}_{0}<n / 2 \quad \Rightarrow \quad \mathbf{b}(k)=\mathbf{0}_{n}, \quad \forall k \geq k^{*} .
$$

Here we only consider the strict majority scenarios. Besides the undirected synchronous network with fixed topology assumption, here it is also assumed that each node can only communicate to its one-hop neighbors and can only transmit its current binary vote. It is important to note that when there is no binary communication constraint, i.e., the nodes are allowed to communicate real numbers rather than binary values, then binary consensus can be treated as a typical consensus problem and there exist numerous algorithms similar to those proposed by Olfati-Saber and Murray [17]-[21]. For example, a consensus protocol of the form

$$
\widehat{\mathbf{x}}(k+1)=\widehat{\mathbf{x}}(k)-\alpha \mathcal{L} \widehat{\mathbf{x}}(k), \quad \widehat{\mathbf{x}}(1)=\mathbf{b}_{0}, \quad k \geq 1,
$$

would guarantee that each node's internal state $\widehat{\mathbf{x}}(k)$ will converge to the average of the initial vote $\mathbf{b}_{0}$. Here $0<\alpha<1$ is a positive scalar value and $\mathcal{L}$ indicates the network Laplacian. The constant $\alpha$ is selected such that the $n-1$ eigenvalues of $\left(I_{n \times n}-\alpha \mathcal{L}\right)$ are less than one and the $n^{\text {th }}$ eigenvalue is one.

Lemma 1: For a connected undirected network, the discrete-time consensus protocol (1) guarantees that

i) the network state $\widehat{\mathbf{x}}(k)$ converges to the average of the initial state $\mathbf{b}_{0}$ at an exponential rate, i.e.,

$$
\left\|\widehat{\mathbf{x}}(k)-\frac{1}{n} \mathbf{1}_{n} \mathbf{1}_{n}^{T} \mathbf{b}_{0}\right\| \leq \kappa \lambda_{\min }\left(I_{n \times n}-\alpha \mathcal{L}\right)^{k},
$$

where $\kappa$ is a positive constant, $\mathbf{1}_{n}$ is an $n$-dimensional vector of ones and $\lambda_{\min }(\cdot)$ indicates the minimum eigenvalue; and ii) for all $k \geq 1$, we have $\mathbf{1}_{n}^{T} \widehat{\mathbf{x}}(k)=\mathbf{1}_{n}^{T} \mathbf{b}_{0}$. 
Proof: Detailed proof is not given due to space constraints, but it easily follows from considering the eigenvalues and eigenvectors of $\left(I_{n \times n}-\alpha \mathcal{L}\right)$.

After the nodes have reached consensus on $\frac{1}{n} \mathbf{1}_{n} \mathbf{1}_{n}^{T} \mathbf{b}_{0}$, i.e., when $\mathcal{L} \widehat{\mathbf{x}}(k)=\mathbf{0}$, majority voting can be achieved if we select

$$
\mathbf{b}(k)= \begin{cases}\mathbf{0}_{n}, & \text { if } \widehat{x}_{i}(k)<\frac{1}{2}, \forall i \in\{1, \ldots, n\} \\ \mathbf{1}_{n}, & \text { if } \widehat{x}_{i}(k)>\frac{1}{2}, \forall i \in\{1, \ldots, n\} .\end{cases}
$$

Binary consensus under binary communication constraint is much more difficult but an important problem. However, currently it is believed that a networked set of agents holding binary opinions are unable to compute the majority opinion by means of local binary interactions [3]. Contrary to popular belief, here we propose a binary consensus protocol, which would allow the nodes to reach consensus on majority opinion by means of local binary communications.

\section{MAIN Results}

The initial binary state of the network consisting of $n$ nodes is denoted as $\mathbf{x}_{0}$. Let $\mathcal{A}$ represent the adjacency matrix, $\mathcal{D}$ the degree matrix, and $\mathcal{L}$ the Laplacian of the network under consideration. Most of the existing work on binary consensus relies on local majority based decision making [9]-[14]. That is, the local vote at node $i, b_{i}(k)$, is selected to be 1 if the local average is greater than $1 / 2$, and $b_{i}(k)$ is selected to be 0 if the local average is less than $1 / 2$. The local average of $\mathbf{x}_{0}$ at each node can be calculated as $S \mathbf{x}_{0}$, where $S$ is given by

$$
S \triangleq\left[\mathcal{D}+I_{n \times n}\right]^{-1}\left[\mathcal{A}+I_{n \times n}\right] .
$$

On the other hand, the traditional consensus protocols, such as those proposed by Olfati-Saber and Murray [17]-[21], rely on the local disagreement. Therefore, the proposed binary consensus protocol is based on both the local majority as well as the local disagreement. The proposed solution makes use of an internal state, which indicates the learning process occurring at each node and the internal state depends on the initial state, local average, and local disagreement. Each node then votes based on the time average of its internal state. Details of the proposed protocol follow.

\section{A. Majority Consensus Protocol}

Let $\mathbf{x}(k) \in \mathbb{R}^{n}$ denote the internal state of the network at time $k$, and $\widehat{\mathbf{b}}(k)$ the network vote at time $k$, where $\widehat{b}_{i}(k) \in\{0,1\}$, for all $i \in\{1,2, \ldots, n\}$ and $k \geq 1$. Note that here we assume that the nodes are only allowed to communicate to their one-hop neighbors and they can only transmit the signal $\widehat{\mathbf{b}}(k)$. The goal is to have the entire network reach consensus on the majority of the initial network state, i.e., $\exists k^{*}: \forall \ell \geq k^{*}$,

$$
\widehat{\mathbf{b}}(\ell)= \begin{cases}\mathbf{0}_{n}, & \text { if } \mathbf{x}_{0}^{T} \mathbf{x}_{0}<\frac{n}{2} \\ \mathbf{1}_{n}, & \text { if } \mathbf{x}_{0}^{T} \mathbf{x}_{0}>\frac{n}{2} .\end{cases}
$$

Here we only consider the strict majority scenarios. Each node bases its vote on the time average of its internal state and the internal state is updated based on the following rule:

$$
\mathbf{x}(k+1)=\alpha \mathbf{x}(k)+M \widehat{\mathbf{b}}(k), \quad \mathbf{x}(1)=\mathbf{x}_{0} .
$$

Here $0<\alpha<1$ is a positive scalar value close to 1 and the matrix $M$ is defined as

$$
M=(1-\alpha) S-\gamma \mathcal{L},
$$

where $\gamma$ is a positive scalar that weights or discounts the local disagreement. The first term $S$ accounts for the local average of the network vote and the second term accounts for the local disagreement. Without the local average term, the protocol given in (5) is very similar to the conventional discrete time consensus protocol. The gain $\alpha$ assures the stability of the system and the gain $(1-\alpha)$ guarantees that the consensus scenarios $\mathbf{x}(k)=\mathbf{1}_{n}$ and $\mathbf{x}(k)=\mathbf{0}_{n}$ are stable equilibria. The gain $\alpha$ is selected to be close to one because a large $\alpha<1$ guarantees the stability as well as assures that the node states are only sensitive to persistent disagreement and the initial votes are not easily altered/forgotten.

Let $\mathbf{y}(k) \in \mathbb{R}^{n}$ denote the time average of the internal state of the network at time $k$, i.e., $\mathbf{y}(k)=\frac{1}{k} \sum_{i=1}^{k} \mathbf{x}(i)$. Now a recursive equation for $\mathbf{y}(k)$ can be written as

$$
\mathbf{y}(k+1)=\frac{k}{k+1} \mathbf{y}(k)+\frac{\alpha}{k+1} \mathbf{x}(k)+\frac{1}{k+1} M \widehat{\mathbf{b}}(k),
$$

where $\mathbf{y}(1)=\mathbf{x}_{0}$. The individual nodes update their vote based on the following decision function $Q(\cdot)$ :

$$
\widehat{\mathbf{b}}(k)=Q(\mathbf{y}(k))=\left[\begin{array}{c}
\min \left\{\left\lfloor\frac{\left.\mid y_{1}(k)\right\rfloor}{0.5}\right\rfloor, 1\right\} \\
\vdots \\
\min \left\{\left\lfloor\frac{\left.\mid y_{n}(k)\right\rfloor}{0.5}\right\rfloor, 1\right\}
\end{array}\right]=\left[\begin{array}{c}
\phi\left(y_{1}(k)\right) \\
\vdots \\
\phi\left(y_{n}(k)\right)
\end{array}\right]
$$

where $\lfloor\cdot\rfloor$ is the floor function and $\phi(\cdot)$ is the nonlinear function defined as

$$
\phi(\cdot)=\min \left\{\left\lfloor\frac{|\cdot|}{0.5}\right\rfloor, 1\right\} .
$$

Thus the node $i$ votes one if $\left|y_{i}(k)\right|$ is greater than or equal to 0.5 and zero otherwise. The decision function depends on the time average of the internal state rather than the internal state itself because the time average acts like an integral and suppresses any sudden variations in $\mathbf{x}(k)$. This makes sure that the nodes alter their votes only based on persistent changes in the internal state.

Therefore, the proposed binary consensus protocol involves an internal state $\mathbf{x}(k)$, its time average $\mathbf{y}(k)$, and the network vote $\widehat{\mathbf{b}}(k)$, i.e.,

$$
\begin{aligned}
\mathbf{x}(k+1) & =\alpha \mathbf{x}(k)+M \widehat{\mathbf{b}}(k), \\
\mathbf{y}(k+1) & =\frac{1}{k+1}(k \mathbf{y}(k)+\alpha \mathbf{x}(k)+M \widehat{\mathbf{b}}(k)), \\
\widehat{\mathbf{b}}(k) & =Q(\mathbf{y}(k)),
\end{aligned}
$$

with $\mathbf{x}(1)=\mathbf{y}(1)=\widehat{\mathbf{b}}(1)=\mathbf{x}_{0}$ and the decision function $Q(\cdot)$ is given in (8).

\section{B. Scalar Conditions for Majority Consensus}

In this subsection, scalar conditions equivalent to the majority consensus conditions given in (4) are derived.

$$
\begin{aligned}
\text { Let } \boldsymbol{\beta}(k) & =\sum_{i=1}^{k} \alpha^{k-i} \widehat{\mathbf{b}}(i) . \text { Thus (5) and (7) can be written as } \\
\mathbf{x}(k+1) & =\alpha^{k} \mathbf{x}_{0}+M \boldsymbol{\beta}(k), \text { and } \\
\mathbf{y}(k+1) & =\frac{k}{k+1} \mathbf{y}(k)+\frac{\alpha^{k}}{k+1} \mathbf{x}_{0}+\frac{1}{k+1} M \boldsymbol{\beta}(k) .
\end{aligned}
$$

Remark 1: Equation (10) clearly indicates that $\alpha$ dictates how fast the initial vote is forgotten within a node.

Note that $\boldsymbol{\beta}(k+1)$ can be written recursively as

$$
\boldsymbol{\beta}(k+1)=\alpha \boldsymbol{\beta}(k)+\widehat{\mathbf{b}}(k+1), \quad \boldsymbol{\beta}(1)=\mathbf{x}_{0} .
$$

Let $\mathbf{q}(k)$ denote $\mathbf{y}(k+1)$ so as to simplify notation. Thus (12) can be rewritten as

$$
\begin{aligned}
& \boldsymbol{\beta}(k+1)=\alpha \boldsymbol{\beta}(k)+Q(\mathbf{q}(k)), \quad \text { or } \\
& \beta_{i}(k+1)=\alpha \beta_{i}(k)+\phi\left(q_{i}(k)\right), \quad \forall i \in\{1, \ldots, n\} .
\end{aligned}
$$

Now $\mathbf{q}(k+1)$ can be written as

$$
\begin{aligned}
\mathbf{q}(k+1)=\frac{k+1}{k+2} \mathbf{q}(k) & +\frac{\alpha^{k+1}}{k+2} \mathbf{x}_{0}+\frac{\alpha}{k+2} M \boldsymbol{\beta}(k) \\
& +\frac{1}{k+2} M Q(\mathbf{q}(k)) .
\end{aligned}
$$


Thus the proposed binary consensus protocol in (9) can be rewritten in terns of $\boldsymbol{\beta}(k)$ and $\mathbf{q}(k)$ as

$$
\begin{aligned}
\overline{\boldsymbol{\beta}(k+1)}=\alpha \boldsymbol{\beta}(k)+Q(\mathbf{q}(k)), & \\
\mathbf{q}(k+1)=\frac{k+1}{k+2} \mathbf{q}(k) & +\frac{\alpha^{k+1}}{k+2} \mathbf{x}_{0}+\frac{\alpha}{k+2} M \boldsymbol{\beta}(k) \\
& +\frac{1}{k+2} M Q(\mathbf{q}(k)) .
\end{aligned}
$$

Define

$$
\begin{aligned}
\bar{\beta}(k) & =\mathbf{1}_{n}^{T} M \boldsymbol{\beta}(k), \quad \text { and } \\
\bar{q}(k) & =\mathbf{1}_{n}^{T} \mathbf{q}(k) .
\end{aligned}
$$

In what follows, scalar conditions equivalent to the majority consensus conditions given in (4) are derived in terms of $\bar{\beta}(k)$ and $\bar{q}(k)$. First, we prove that $\lim \bar{\beta}(k)=n$ implies that there exists a $k^{*}$

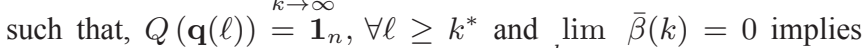
that there exists a $k^{*}$ such that, $Q(\mathbf{q}(\ell)) \stackrel{k \rightarrow \infty}{=} \mathbf{0}_{n}, \forall \ell \geq k^{*}$.

Lemma 2: The limit $\lim _{k \rightarrow \infty} \bar{\beta}(k)=n$ holds if and only if there exists a $k^{*}$ such that, $Q(\mathbf{q}(\ell))=\mathbf{1}_{n}, \forall \ell \geq k^{*}$. Similarly, the limit $\lim _{k \rightarrow \infty} \bar{\beta}(k)=0$ holds if and only if there exists a $k^{*}$ such that,

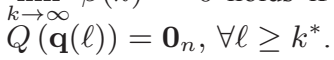

Proof: Omitted due to space restrictions.

Define $\boldsymbol{\zeta}(k)=\sum_{i=1}^{k} \widehat{\mathbf{b}}(i)=\sum_{j=0}^{k-1} Q(\mathbf{q}(j))$. Now $\mathbf{q}(k)$ can be written in terms of $\boldsymbol{\zeta}(k)$ as

$$
\begin{aligned}
\mathbf{q}(k)=\frac{1}{k+1}\left\{\left(\frac{1-\alpha^{k+1}}{1-\alpha}\right) \mathbf{x}_{0}\right. & +\frac{1}{1-\alpha} M \boldsymbol{\zeta}(k) \\
& \left.-\frac{\alpha}{1-\alpha} M \boldsymbol{\beta}(k)\right\} .
\end{aligned}
$$

Note that for all $i \in\{1, \ldots, n\}, \zeta_{i}(k)$ is upper and lower bounded as $0 \leq \zeta_{i}(k) \leq k$.

Proposition 1: The limit $\lim _{k \rightarrow \infty} \mathbf{q}(k)=\mathbf{1}_{n}$ holds if and only if $\lim _{k \rightarrow \infty} \frac{1}{k+1} \boldsymbol{\zeta}(k)=\mathbf{1}_{n}$. Similarly, limit $\lim _{k \rightarrow \infty} \mathbf{q}(k)=\mathbf{0}_{n}$ holds if and only if $\lim _{k \rightarrow \infty} \frac{1}{k+1} \boldsymbol{\zeta}(k)=\mathbf{0}_{n}$

Proof: Omitted due to space restrictions.

Now we prove that $\lim \bar{q}(k)=n$ implies that there exists a

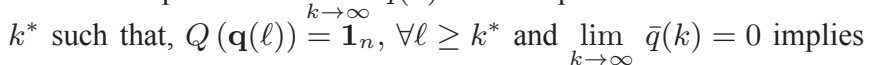
that there exists a $k^{*}$ such that, $Q(\mathbf{q}(\ell))=\mathbf{0}_{n}, \forall \ell \geq k^{*}$.

Lemma 3: The limit $\lim _{k \rightarrow \infty} \bar{q}(k)=n$ holds if and only if there

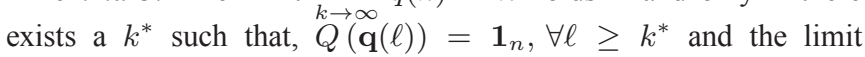
$\lim _{k \rightarrow \infty} \bar{q}(k)=0$ holds if and only if then there exists a $k^{*}$ such that, $Q(\mathbf{q}(\ell))=\mathbf{0}_{n}, \forall \ell \geq k^{*}$.

Proof: Omitted due to space restrictions.

From (12) and (15) $\bar{\beta}(k+1)$ and $\bar{q}(k+1)$ can be written as

$$
\begin{aligned}
\bar{\beta}(k+1) & =\alpha \bar{\beta}(k)+u(k), \\
\bar{q}(k+1)=\frac{k+1}{k+2} \bar{q}(k)+\frac{\alpha^{k+1}}{k+2} & x_{0}+\frac{\alpha}{k+2} \bar{\beta}(k) \\
& +\frac{1}{k+2} u(k),
\end{aligned}
$$

where $x_{0}=\mathbf{1}_{n}^{T} \mathbf{x}_{0}$ and $u(k)=\mathbf{1}_{n}^{T} M Q(\mathbf{q}(k))$. Now based on Lemma 2 and Lemma 3, the majority consensus condition in (4) can be rewritten as

$$
\lim _{k \rightarrow \infty}\left[\begin{array}{l}
\bar{q}(k) \\
\bar{\beta}(k)
\end{array}\right]= \begin{cases}\mathbf{0}_{2}, & \text { if } \mathbf{x}_{0}^{T} \mathbf{x}_{0}<\frac{n}{2} \\
n \times \mathbf{1}_{2}, & \text { if } \mathbf{x}_{0}^{T} \mathbf{x}_{0}>\frac{n}{2} .\end{cases}
$$

Thus, the majority consensus protocol can be proven if the above condition holds for the decision rule given in (8).

\section{Sector Bound Condition}

The nonlinear function $\phi(\cdot)$ used in the decision function $Q(\cdot)$ is sector bounded [22].

Definition 1: A function $\phi: \mathbb{R} \rightarrow \mathbb{R}$ is said to be in sector $\left[l_{b}, u_{b}\right]$ if and only if for all $q \in \mathbb{R}$,

$$
\left|\phi(q)-\frac{u_{b}+l_{b}}{2} q\right| \leq \frac{u_{b}-l_{b}}{2}|q| .
$$

Based on the definition, the nonlinear function $\phi(\cdot)$ considered here is in sector $[-2,2]$ and satisfies the following sector bound inequality

$$
\left(\phi\left(q_{i}(k)\right)-2\left|q_{i}(k)\right|\right) \leq 0, \forall k \geq 1, i \in\{1, \ldots, n\} .
$$

Note that $\mathbf{1}_{n}^{T} M Q(\mathbf{q}(k)) \leq \mathbf{1}_{n}^{T} M \mathbf{1}_{n}=(1-\alpha) n$, and each element of the row vector $\mathbf{1}_{n}^{T} M$ are $\geq 0$. Therefore,

$$
\left(\mathbf{1}_{n}^{T} M\right)_{i} \phi\left(q_{i}(k)\right)-2\left(\mathbf{1}_{n}^{T} M\right)_{i}\left|q_{i}(k)\right| \leq 0,
$$

where $\left(\mathbf{1}_{n}^{T} M\right)_{i}$ indicates the $i^{\text {th }}$ element of $\left(\mathbf{1}_{n}^{T} M\right)$. Now summing over $i$ yields $\sum_{i=1}^{n}\left(\mathbf{1}_{n}^{T} M\right)_{i} \phi\left(q_{i}(k)\right)=\mathbf{1}_{n}^{T} M Q(\mathbf{q}(k))$, and $\sum_{\substack{i=1 \\ \text { have }}}^{n}\left|\left(\mathbf{1}_{n}^{T} M\right)_{i} q_{i}(k)\right|=\mathbf{1}_{n}^{T} M$ abs $\{\mathbf{q}(k)\}$. From (19) and (6), we

$$
\begin{aligned}
\operatorname{abs}\{\mathbf{q}(k)\}= & \frac{1}{k+1} \text { abs }\left\{\left(\frac{1-\alpha^{k+1}}{1-\alpha}\right) \mathbf{x}_{0}+\right. \\
& \left.\frac{1}{1-\alpha} M \boldsymbol{\zeta}(k)-\frac{\alpha}{1-\alpha} M \boldsymbol{\beta}(k)\right\}, \\
= & \frac{1}{k+1} \operatorname{abs}\left\{\left(\frac{1-\alpha^{k+1}}{1-\alpha}\right) \mathbf{x}_{0}+\right. \\
& \left.S(\boldsymbol{\zeta}(k)-\alpha \boldsymbol{\beta}(k))-\frac{\gamma}{1-\alpha} \mathcal{L}(\boldsymbol{\zeta}(k)-\alpha \boldsymbol{\beta}(k))\right\} .
\end{aligned}
$$

Note $\quad \mathbf{0}_{n} \leq \frac{1}{k+1}(\boldsymbol{\zeta}(k)-\alpha \boldsymbol{\beta}(k)) \leq \mathbf{1}_{n}$, and abs $\left\{\frac{1}{k+1} \mathcal{L}(\boldsymbol{\zeta}(k)-\alpha \boldsymbol{\beta}(k))\right\} \leq \operatorname{diag}\{D\}$. Thus using (19),

$$
\begin{aligned}
& \mathbf{1}_{n}^{T} M \operatorname{abs}\{\mathbf{q}(k)\} \leq \mathbf{1}_{n}^{T} M \frac{1}{k+1} \text { abs }\left\{\left(\frac{1-\alpha^{k+1}}{1-\alpha}\right) \mathbf{x}_{0}+\right. \\
& S(\boldsymbol{\zeta}(k)-\alpha \boldsymbol{\beta}(k))\}+\mathbf{1}_{n}^{T} M \frac{1}{k+1} \text { abs }\left\{\frac{\gamma}{1-\alpha} \mathcal{L}(\boldsymbol{\zeta}(k)-\alpha \boldsymbol{\beta}(k))\right\} \\
& \leq \max \left\{\mathbf{1}_{n}^{T} M\right\} \bar{q}(k)+\frac{\gamma}{1-\alpha} \mathbf{1}_{n}^{T} M \operatorname{diag}\{D\} .
\end{aligned}
$$

The first term in the last inequality follows from the fact that for a vector $\mathbf{e} \geq \mathbf{0}, \mathbf{1}_{n}^{T} M \mathbf{e} \leq \max \left\{\mathbf{1}_{n}^{T} M\right\} \mathbf{1}_{n}^{T} \mathbf{e}$ and $\mathbf{1}_{n}^{T}$ is a left eigenvector of $\mathcal{L}$, associated with the eigenvalue 0 . Let $\varrho=$ $\frac{\gamma}{1-\alpha} \mathbf{1}_{n}^{T} M \operatorname{diag}\{D\}$ and $\rho^{*}=\max \left\{\mathbf{1}_{n}^{T} M\right\}$. Now we have

$$
u(k) \leq 2 \rho^{*} \bar{q}(k)+2 \varrho .
$$

After multiplying both sides by $u(s)$, we have the following quadratic inequality:

$$
u^{2}(k) \leq 2 \rho^{*} \bar{q}(k) u(k)+2 \varrho u(k) .
$$

Let $\mathbf{z}(k)=\left[\begin{array}{lllll}\bar{\beta}(k) & \bar{q}(k) & u(k) & x_{0} & \varrho\end{array}\right]^{T}$, now the above inequality can be written as $\mathbf{z}^{T}(k) \Sigma_{1} \mathbf{z}(k) \leq 0$, where

$$
\Sigma_{1}=\left[\begin{array}{ccccc}
0 & 0 & 0 & 0 & 0 \\
0 & 0 & -\rho^{*} & 0 & 0 \\
0 & -\rho^{*} & 1 & 0 & -1 \\
0 & 0 & 0 & 0 & 0 \\
0 & 0 & -1 & 0 & 0
\end{array}\right] .
$$

\section{Convergence Analysis}

For the convergence analysis, we consider the appended vector $\mathbf{z}(k)$. Since $\mathbf{z}(k)$ is five-dimensional and so far we only have the dynamic expression for two of its elements; in order to facilitate the convergence analysis, the following three dummy variables are 
considered in addition to $\bar{q}(k)$ and $\bar{\beta}(k)$ :

$$
\begin{aligned}
\mu(k) & =\bar{\beta}(k)-\frac{\alpha^{k}}{\varrho} \varrho, \\
\eta(k) & =\frac{1}{n} \bar{\beta}(k)+\bar{q}(k), \\
\theta(k) & =\bar{q}(k)-\alpha^{k} x_{0} .
\end{aligned}
$$

Define $\boldsymbol{z}(k)=\left[\begin{array}{lllll}\bar{\beta}(k) & \bar{q}(k) & \mu(k) & \eta(k) & \theta(k)\end{array}\right]$. Thus, $\boldsymbol{z}(k)=$ $\Gamma_{1}(k) \mathbf{z}(k)$ and $\boldsymbol{z}(k+1)=\Gamma_{2}(k) \mathbf{z}(k)$, where

$$
\begin{gathered}
\Gamma_{1}(k)=\left[\begin{array}{ccccc}
1 & 0 & 0 & 0 & 0 \\
0 & 1 & 0 & 0 & 0 \\
1 & 0 & 0 & 0 & -\frac{\alpha^{k}}{\varrho} \\
\frac{1}{n} & 1 & 0 & 0 & 0 \\
0 & 1 & 0 & -\alpha^{k} & 0
\end{array}\right] \text { and } \Gamma_{2}(k)= \\
{\left[\begin{array}{ccccc}
\alpha & 0 & 1 & 0 & 0 \\
\frac{\alpha}{k+2} & \frac{k+1}{k+2} & \frac{1}{k+2} & \frac{\alpha^{k+1}}{k+2} & 0 \\
\alpha & 0 & 1 & 0 & -\frac{\alpha^{k+1}}{\varrho} \\
\frac{\alpha}{n}+\frac{\alpha}{k+2} & \frac{k+1}{k+2} & \frac{1}{n}+\frac{1}{k+2} & \frac{\alpha^{k+1}}{k+2} & 0 \\
\frac{\alpha}{k+2} & \frac{k+1}{k+2} & \frac{1}{k+2} & -\alpha^{k+1}+\frac{\alpha^{k+1}}{k+2} & 0
\end{array}\right]}
\end{gathered}
$$

Since for all $k \geq 1, \bar{\beta}(k) \leq n, \bar{q}(k) \leq n$, and $u(k) \leq(1-\alpha) n$, the following inequalities are valid for all $k$ :

$$
\begin{aligned}
& \bar{\beta}^{2}(k)-\bar{\beta}(k) x_{0}-\left(n-x_{0}\right) \bar{\beta}(k) \leq 0, \\
& \bar{q}^{2}(k)-\bar{q}(k) n \leq 0, \\
& u^{2}(k)-u(k) \frac{((1-\alpha) n)}{x_{0}} x_{0} \leq 0, \\
& \frac{1}{\varrho} \varrho^{2}-\frac{1.5}{x_{0}} \varrho x_{0} \leq 0 .
\end{aligned}
$$

The above inequalities can be written in matrix form as

$$
\mathbf{z}^{T}(k) \Sigma_{\imath} \mathbf{z}(k) \leq 0, \quad \text { for } \quad \imath \in\{2,3,4,5\},
$$

where

$$
\begin{aligned}
\Sigma_{2} & =\left[\begin{array}{cccc}
1 & \mathbf{0}_{1 \times 2} & -\frac{1}{2} & -\frac{n-x_{0}}{2 \varrho} \\
\mathbf{0}_{2 \times 1} & 0_{2 \times 2} & \mathbf{0}_{2 \times 1} & \mathbf{0}_{2 \times 1} \\
-\frac{1}{2} & \mathbf{0}_{1 \times 2} & 0 & 0 \\
-\frac{n-x_{0}}{2 \varrho} & \mathbf{0}_{1 \times 2} & 0 & 0
\end{array}\right], \\
\Sigma_{3} & =\left[\begin{array}{cccc}
0 & 0 & \mathbf{0}_{1 \times 2} & 0 \\
0 & 1 & \mathbf{0}_{1 \times 2} & -\frac{n}{2 \varrho} \\
\mathbf{0}_{2 \times 1} & \mathbf{0}_{2 \times 1} & 0_{2 \times 2} & \mathbf{0}_{2 \times 1} \\
0 & -\frac{n}{2 \varrho} & \mathbf{0}_{1 \times 2} & 0
\end{array}\right], \\
\Sigma_{4} & =\left[\begin{array}{cccc}
0_{2 \times 2} & \mathbf{0}_{2 \times 1} & \mathbf{0}_{2 \times 1} & \mathbf{0}_{2 \times 1} \\
\mathbf{0}_{1 \times 2} & 1 & -\frac{((1-\alpha) n)}{2 x_{0}} & 0 \\
\mathbf{0}_{1 \times 2} & -\frac{((1-\alpha) n)}{2 x_{0}} & 0 & 0 \\
\mathbf{0}_{1 \times 2} & 0 & 0 & 0
\end{array}\right], \\
\Sigma_{5} & =\left[\begin{array}{ccc}
0_{3 \times 3} & \mathbf{0}_{3 \times 1} & \mathbf{0}_{3 \times 1} \\
\mathbf{0}_{1 \times 3} & 0 & -\frac{1.5}{2 x_{0}} \\
\mathbf{0}_{1 \times 3} & -\frac{1.5}{2 x_{0}} & \frac{1}{\varrho}
\end{array}\right] .
\end{aligned}
$$

Now construct a positive definite function of following form

$V(k)=\boldsymbol{z}^{T}(k) P \boldsymbol{z}(k)=\left\{\Gamma_{1}(k) \mathbf{z}(k)\right\}^{T} P\left\{\Gamma_{1}(k) \mathbf{z}(k)\right\}, \quad$ (34) where $P \in \mathbb{R}^{5 \times 5}$ is positive definite square matrix. Now $\Delta V(k)=$ $V(k+1)-V(k)$ can be calculated as

$$
\Delta V(k)=\mathbf{z}^{T}(k)\left\{\Gamma_{2}^{T}(k) P \Gamma_{2}(k)-\Gamma_{1}^{T}(k) P \Gamma_{1}(k)\right\} \mathbf{z}(k) .
$$

1) Majority Scenario 1: Here we first consider the "0" majority scenario, i.e., $x_{0}<n / 2$ or $x_{0} \leq \frac{n-1}{2}$. The condition $x_{0} \leq \frac{n-1}{2}$ can be written in quadratic form as

$$
\mathbf{z}^{T}(k) \Sigma_{6} \mathbf{z}(k) \leq 0,
$$

where $\Sigma_{6}=\left[\begin{array}{ccc}0_{3 \times 3} & \mathbf{0}_{3 \times 1} & \mathbf{0}_{3 \times 1} \\ \mathbf{0}_{1 \times 3} & \frac{1}{x_{0}} & -\frac{\left(n-1-x_{0}\right)}{2 x_{0} \varrho} \\ \mathbf{0}_{1 \times 3} & -\frac{\left(n-1-x_{0}\right)}{2 x_{0} \varrho} & 0\end{array}\right]$.

Thus, for the proposed approach, majority consensus for the " 0 " majority scenario can be reached if

$$
\mathbf{z}^{T}(k) \Sigma_{6} \mathbf{z}(k) \leq 0 \Rightarrow \lim _{k \rightarrow \infty} \Gamma_{1}(k) \mathbf{z}(k)=\mathbf{0}_{5},
$$

whenever $\mathbf{z}(k)$ satisfies the quadratic inequalities $\mathbf{z}^{T}(k) \Sigma_{\imath} \mathbf{z}(k) \leq$ 0 , for $\imath \in\{1,2,3,4,5\}$.

Note that the positive definite function, $V(k)$, in (34) is always upper bounded and therefore, if there exists a $k^{*}$ such that, if for all $k \geq k^{*}, \Delta V(k)<0$, then it can be concluded that $\lim _{k \rightarrow \infty} \Gamma_{1}(k) \mathbf{z}(k)=\mathbf{0}_{5}$. Therefore, we would like the nonpositivity of a set of quadratic forms, $\left\{\mathbf{z}^{T}(k) \Sigma_{1} \mathbf{z}(k), \mathbf{z}^{T}(k) \Sigma_{2} \mathbf{z}(k), \ldots, \mathbf{z}^{T}(k) \Sigma_{6} \mathbf{z}(k)\right\}$, to imply the negativity of $\mathbf{z}^{T}(k)\left\{\Gamma_{2}^{T}(k) P \Gamma_{2}(k)-\Gamma_{1}^{T}(k) P \Gamma_{1}(k)\right\} \mathbf{z}(k)$.

Based on the (lossy) S-procedure [22], a simple sufficient criteria that would guarantee the negativity of a quadratic term given the nonpositivity of a set of quadratic forms can be obtained as follows:

If there exist $\tau_{1}(k), \tau_{2}(k), \ldots, \tau_{6}(k) \geq 0$ such that for all $k \geq$ $k^{*},\left\{\Gamma_{2}^{T}(k) P \Gamma_{2}(k)-\Gamma_{1}^{T}(k) P \Gamma_{1}(k)\right\}-\sum_{i=1}^{6} \tau_{i}(k) \Sigma_{i}<0$, then $\forall \mathbf{z}(k)$,

$$
\begin{aligned}
& \mathbf{z}^{T}(k) \Sigma_{1} \mathbf{z}(k) \leq 0, \ldots, \mathbf{z}^{T}(k) \Sigma_{6} \mathbf{z}(k) \leq 0 \Rightarrow \\
& \quad \mathbf{z}^{T}(k)\left\{\Gamma_{2}^{T}(k) P \Gamma_{2}(s)-\Gamma_{1}^{T}(k) P \Gamma_{1}(k)\right\} \mathbf{z}(k)<0 .
\end{aligned}
$$

Theorem 1: For an undirected connected network of $n$ nodes with initial binary state $\mathbf{x}_{0}$ such that, $\mathbf{1}_{n}^{T} \mathbf{x}_{0}<n / 2$, the decision function $Q(\cdot)$ given in (8) guarantees that the nodes asymptotically reach consensus to $\mathbf{0}_{n}$, if there exists a $k^{*} \geq 1$, a positive-definite symmetric matrix $P$, and sequences $\tau_{1}(k), \tau_{2}(k), \ldots, \tau_{6}(k) \geq 0$ such that, for all $k \geq k^{*}$,

$$
\left\{\Gamma_{2}^{T}(k) P \Gamma_{2}(k)-\Gamma_{1}^{T}(k) P \Gamma_{1}(k)\right\}-\sum_{i=1}^{6} \tau_{i}(k) \Sigma_{i}<0 .
$$

Proof: The proof follows directly from considering a Lyapunov function, $V(k)$, as given in (34)

2) Majority Scenario 2: Now we consider the "1" majority scenario, i.e., $x_{0}>n / 2$ or $x_{0} \geq \frac{n+1}{2}$. The condition $x_{0} \geq \frac{n+1}{2}$ can be written in quadratic form as

where $\Sigma_{6}^{\prime}=\left[\begin{array}{ccc}\mathbf{z}_{3 \times 3} & \mathbf{0}_{3 \times 1}(k) \Sigma_{6}^{\prime} \mathbf{z}(k) \leq 0, \\ \mathbf{0}_{1 \times 3} & \frac{n+1}{2 x_{0}^{2}} & -\frac{1}{2 \varrho} \\ \mathbf{0}_{1 \times 3} & -\frac{1}{2 \varrho} & 0\end{array}\right]$. Based on (22) and (28), the majority consensus condition for $\boldsymbol{z}(k)$ can be written as

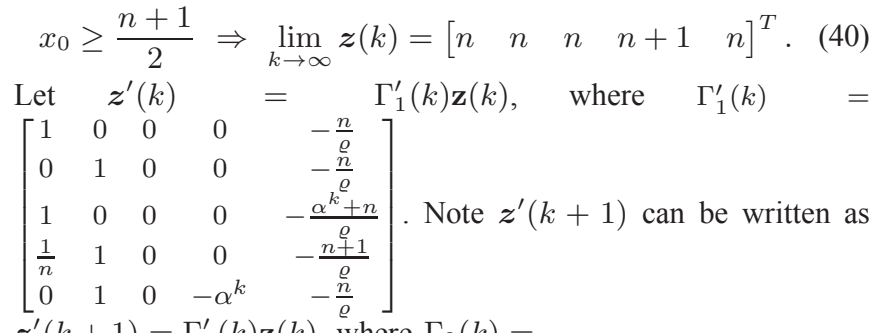
$\boldsymbol{z}^{\prime}(k+1)=\Gamma_{2}^{\prime}(k) \mathbf{z}(k)$, where $\Gamma_{2}(k)=$

$\left[\begin{array}{ccccc}\alpha & 0 & 1 & 0 & -\frac{n}{\varrho} \\ \frac{\alpha}{k+2} & \frac{k+1}{k+2} & \frac{1}{k+2} & \frac{\alpha^{k+1}}{k+2} & -\frac{n}{\varrho} \\ \alpha & 0 & 1 & 0 & -\frac{\alpha^{k+1}+n}{\varrho} \\ \frac{\alpha}{n}+\frac{\alpha}{k+2} & \frac{k+1}{k+2} & \frac{1}{n}+\frac{1}{k+2} & \frac{\alpha^{k+1}}{k+2} & -\frac{n+1}{\varrho} \\ \frac{\alpha}{k+2} & \frac{k+1}{k+2} & \frac{1}{k+2} & -\alpha^{k+1}+\frac{\alpha^{k+1}}{k+2} & -\frac{n}{\varrho}\end{array}\right]$


Thus, for the proposed approach, majority consensus for the "1" majority scenario can be reached if

$$
\mathbf{z}^{T}(k) \Sigma_{6}^{\prime} \mathbf{z}(k) \leq 0 \Rightarrow \lim _{k \rightarrow \infty} \Gamma_{1}^{\prime}(k) \mathbf{z}(k)=\mathbf{0}_{5},
$$

whenever $\mathbf{z}(k)$ satisfies the quadratic inequalities $\mathbf{z}^{T}(k) \Sigma_{\imath} \mathbf{z}(k) \leq$ 0 , for $\imath \in\{1,2,3,4,5\}$.

Now based on the $S$-procedure, it can be concluded that if there exist a $k^{*}$, a positive-definite symmetric matrix $P^{\prime}$, and non-negative scalars $\tau_{1}^{\prime}(k), \tau_{2}^{\prime}(k), \ldots, \tau_{6}^{\prime}(k) \geq 0$ such that, for all $k \geq k^{*}$,

$$
\left\{\Gamma_{2}^{\prime T}(k) P^{\prime} \Gamma_{2}^{\prime}(k)-\Gamma_{1}^{\prime T}(k) P^{\prime} \Gamma_{1}^{\prime}(k)\right\}-\sum_{i=1}^{5} \tau_{i}^{\prime}(k) \Sigma_{i}-\tau_{6}^{\prime}(k) \Sigma_{6}^{\prime}<0,
$$

then $\forall \mathbf{z}(k)$,

$$
\begin{aligned}
& \mathbf{z}^{T}(k) \Sigma_{1} \mathbf{z}(k) \leq 0, \ldots, \mathbf{z}^{T}(k) \Sigma_{6}^{\prime} \mathbf{z}(k) \leq 0 \Rightarrow \\
& \quad \mathbf{z}^{T}(k)\left\{\Gamma_{2}^{\prime T}(k) P^{\prime} \Gamma_{2}^{\prime}(s)-\Gamma_{1}^{\prime T}(k) P^{\prime} \Gamma_{1}^{\prime}(k)\right\} \mathbf{z}(k)<0 .
\end{aligned}
$$

Theorem 2: For an undirected connected network of $n$ nodes with initial binary state $\mathbf{x}_{0}$ such that, $\mathbf{1}_{n}^{T} \mathbf{x}_{0}>n / 2$, the decision function $Q(\cdot)$ given in (8) guarantees that the nodes asymptotically reach consensus to $\mathbf{1}_{n}$, if there exists a $k^{*}$, a positive-definite symmetric matrix $P^{\prime}$, and sequences $\tau_{1}^{\prime}(k), \tau_{2}^{\prime}(k), \ldots, \tau_{6}^{\prime}(k) \geq 0$ such that, for all $k \geq k^{*}$,

$$
\begin{aligned}
\left\{\Gamma_{2}^{\prime T}(k) P^{\prime} \Gamma_{2}^{\prime}(k)-\Gamma_{1}^{\prime T}(k) P^{\prime} \Gamma_{1}^{\prime}(k)\right\} & -\sum_{i=1}^{5} \tau_{i}^{\prime}(k) \Sigma_{i} \\
& -\tau_{6}^{\prime}(k) \Sigma_{6}^{\prime}<0 .
\end{aligned}
$$

Proof: The proof follows directly from considering a Lyapunov function, $V^{\prime}(k)=\boldsymbol{z}^{\prime T}(k) P^{\prime} \boldsymbol{z}^{\prime}(k)$.

\section{NumericAl Results}

Numerical simulations to demonstrate the performance of the proposed binary consensus protocol are given in this section. Consider a line network shown in Fig. 1. For numerical simulations, we select $n=501, \alpha=0.99999$, and $\gamma=0.4$. For the line network consisting of 501 nodes, the numerical simulations are conducted for two different initial binary voting scenarios.

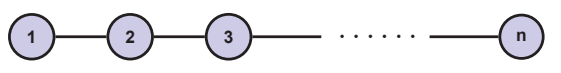

Fig. 1. Line Network

For the first scenario, the initial condition is selected as $\mathbf{x}_{0}=$ $\left[\begin{array}{llll}\mathbf{1}_{125}^{T} & 0 & \mathbf{1}_{125}^{T} & \mathbf{0}_{250}^{T}\end{array}\right]^{T}$. It can be shown that the selected $\alpha$ and $\gamma$ values yield a positive definite matrix $P=10^{4} \times$

$$
\left[\begin{array}{ccccc}
1.5480 & -0.0002 & -1.5480 & -0.0027 & 0.0029 \\
-0.0002 & 2.9094 & -0.0005 & 0.3031 & -3.2110 \\
-1.5480 & -0.0005 & 1.5480 & -0.0031 & 0.0036 \\
-0.0027 & 0.3031 & -0.0031 & 2.9094 & -3.2110 \\
0.0029 & -3.2110 & 0.0036 & -3.2110 & 6.4191
\end{array}\right]
$$

and $\tau_{1}(k), \ldots, \tau_{6}(k)$ as shown in Fig. 2 such that, (38) is satisfied $\forall k \geq 5 \times 10^{5}$ and for the above initial condition $\mathbf{x}_{0}$.

The positive definite matrix $P$ and the scalar quantities $\tau_{1}(k), \ldots, \tau_{6}(k)$ are calculated using the MATLAB LMI toolbox [23]. When $k=1$, the LMI (38) is solved for $P$ and $\tau_{1}(k), \ldots, \tau_{6}(k)$ using the MATLAB LMI toolbox. The positive definite matrix $P$ is held constant for all $k$ while the scalar quantities $\tau_{1}(k), \ldots, \tau_{6}(k)$ are recalculated at each time instant using the LMI toolbox if quantities from the previous time instant, i.e., $\tau_{1}(k-1), \ldots, \tau_{6}(k-1)$ do not satisfy the LMI (38) for the current instant.

Numerical results obtained for the first scenario are given in Fig. 3. Figure 3(a) contains the internal state vector $\mathbf{x}(k)$ obtained for the first scenario. Note that the elements of $\mathbf{x}(k)$ are approaching zero as $k \rightarrow \infty$. Also note the time average of the internal state

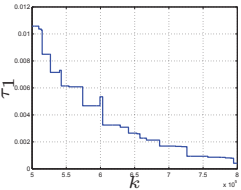

(a) $\tau_{1}(k)$

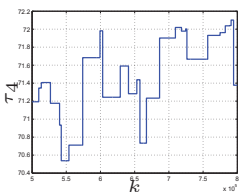

(d) $\tau_{4}(k)$

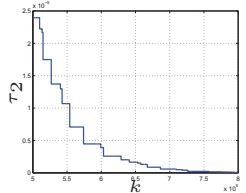

(b) $\tau_{2}(k)$

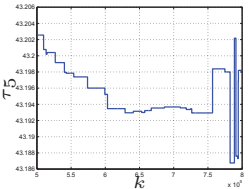

(e) $\tau_{5}(k)$

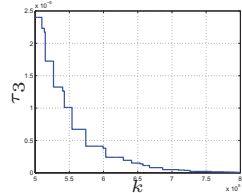

(c) $\tau_{3}(k)$

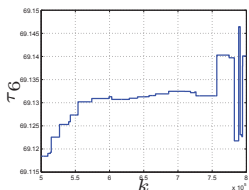

(f) $\tau_{6}(k)$
Fig. 2. Values of $\tau$ 's that satisfies the LMI (38) for a line network with $n=$ $501, \alpha=0.99999, \gamma=0.4$ and initial vote $\mathbf{x}_{0}=\left[\begin{array}{llll}\mathbf{1}_{125}^{T} & 0 & \mathbf{1}_{125}^{T} & \mathbf{0}_{250}^{T}\end{array}\right]^{T}$.

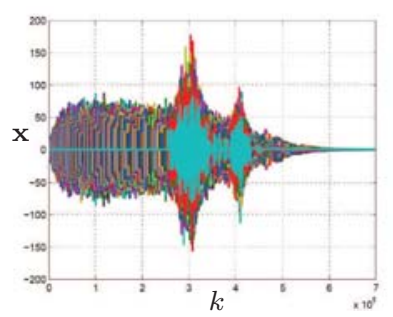

(a) Internal states

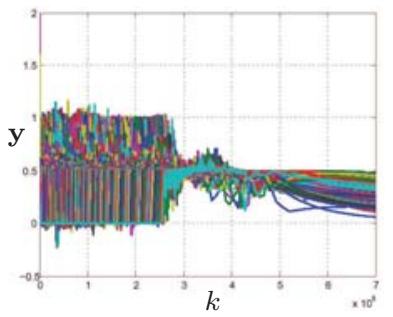

(b) Time average of internal states

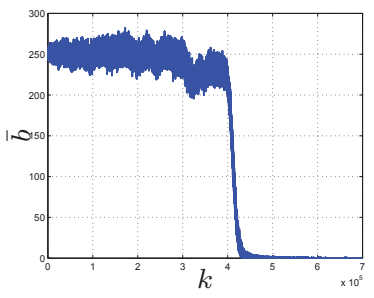

(c) Sum of network votes

Fig. 3. Results obtained for a line network with $n=501$ and initial vote $\mathbf{x}_{0}=\left[\begin{array}{llll}\mathbf{1}_{125}^{T} & 0 & \mathbf{1}_{125}^{T} & \mathbf{0}_{250}^{T}\end{array}\right]^{T}$.

of the network $\mathbf{y}(k)$ given in Fig. 3(b). Figure 3(b) indicates that $\forall k \geq 5 \times 10^{5}$, the elements of $\mathbf{y}(k)$ are asymptotically decaying to zero. Given in Fig. 3(c) is the collective sum of the network vote $\left(\bar{b}(k)=\mathbf{1}_{n}^{T} \mathbf{b}(k)\right)$ and it clearly indicates that the network is able to reach consensus at the majority, 0 .

For the second majority scenario, the initial condition is selected to be $\mathbf{x}_{0}=\left[\begin{array}{llll}\mathbf{1}_{250}^{T} & \mathbf{0}_{125}^{T} & 1 & \mathbf{0}_{125}^{T}\end{array}\right]^{T}$. It can be shown that the selected $\alpha$ and $\gamma$ values could yield a positive definite matrix $P^{\prime}=$ $10^{4} \times$

$$
\left[\begin{array}{ccccc}
1.5380 & 0.0121 & -1.5380 & 0.0117 & -0.0238 \\
0.0121 & 2.9010 & -0.0127 & 0.3052 & -3.2070 \\
-1.5380 & -0.0127 & 1.5380 & -0.0175 & 0.0302 \\
0.0117 & 0.3052 & -0.0175 & 2.9003 & -3.2062 \\
-0.0238 & -3.2070 & 0.0302 & -3.2062 & 6.4146
\end{array}\right]
$$

and $\tau_{1}^{\prime}(k), \ldots, \tau_{6}^{\prime}(k)$ as shown in Fig. 4 such that, (42) is satisfied $\forall k \geq 5 \times 10^{5}$ and for the above initial condition $\mathbf{x}_{0}$.

Numerical results obtained for the second scenario are given in Fig. 5. Figure 5(a) shows that the internal state vector approaches one as $k \rightarrow \infty$. Also, the time average of the internal state of the network given in Fig. 5(b) indicates that $\forall k \geq 5 \times 10^{5}$; the elements of $\mathbf{y}(k)$ are asymptotically approaching one. Given in Fig. 5(c) is the collective sum of the network vote obtained and it clearly indicates that the network is able to reach consensus at the majority, 1. 


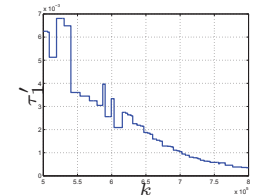

(a) $\tau_{1}^{\prime}(k)$

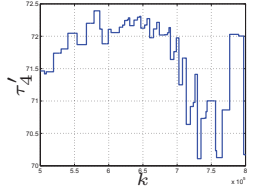

(d) $\tau_{4}^{\prime}(k)$

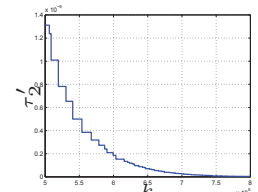

(b) $\tau_{2}^{\prime}(k)$

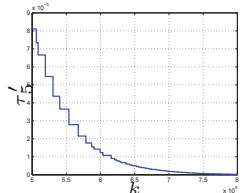

(e) $\tau_{5}^{\prime}(k)$

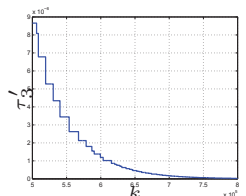

(c) $\tau_{3}^{\prime}(k)$

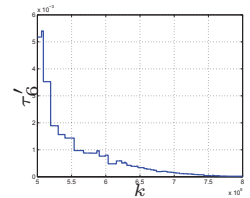

(f) $\tau_{6}^{\prime}(k)$
Fig. 4. Values of $\tau^{\prime}$ 's that satisfies the LMI (42) for a line network with $n=$ $501, \alpha=0.99999, \gamma=0.4$ and initial vote $\mathbf{x}_{0}=\left[\begin{array}{lllll}\mathbf{1}_{250}^{T} & \mathbf{0}_{125}^{T} & 1 & \mathbf{0}_{125}^{T}\end{array}\right]^{T}$.

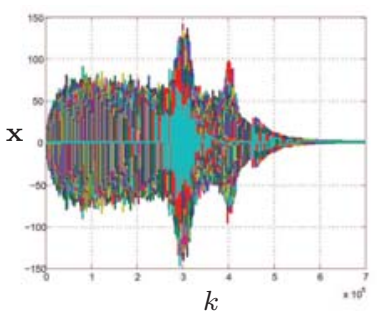

(a) Internal states

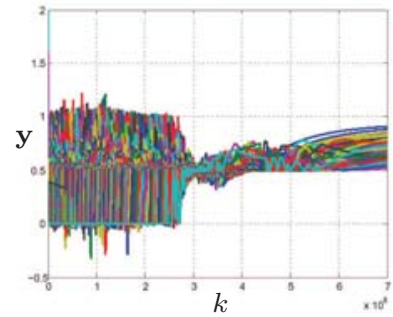

(b) Time average of internal states

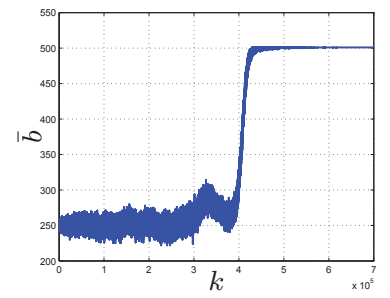

(c) Sum of network votes

Fig. 5. Results obtained for a line network with $n=501$ and initial vote $\mathbf{x}_{0}=\left[\begin{array}{llll}\mathbf{1}_{250}^{T} & \mathbf{0}_{125}^{T} & 1 & \mathbf{0}_{125}^{T}\end{array}\right]^{T}$.

\section{CONCLUSION}

This paper presents the formulation and analysis of a binary consensus protocol for undirected, synchronous networks, which only requires binary communication among one-hop neighbors. The proposed protocol requires the nodes to keep track of an internal state, which accounts for the local disagreement and local average of the network vote. Convergence of the algorithm to the initial global majority is proved using the lossy S-procedure. The binary consensus algorithm contains two design variables: $\alpha$, which dictates how fast the initial vote is forgotten within a node, and $\gamma$, which is a positive scalar what weights or discounts the local disagreement. Numerical results validate the theoretical results. Future work includes analyzing the convergence time of the proposed protocol and modifying the algorithm for different majority scenarios.

\section{REFERENCES}

[1] F. Benezit, P. Thiran, and M. Vetterli, "Interval consensus: From quantized gossip to voting," in Proc. IEEE International Conference on Acoustics, Speech and Signal Processing (ICASSP), Taipei, Taiwan, Apr. 2009, pp. 3661 - 3664.

[2] E. Perron, D. Vasudevan, and M. Vojnovic, "Using three states for binary consensus on complete graphs," in Proc. IEEE International Conference on Computer Communications (INFOCOM), Rio de Janeiro, Brazil, Apr. 2009, pp. 2527-2535.
[3] F. Benezit, P. Thiran, and M. Vetterli, "The distributed multiple voting problem," IEEE Journal of Selected Topics in Signal Processing, vol. 5, no. 4, pp. 791-804, Aug. 2011.

[4] M. Draief and M. Vojnovic, "Convergence speed of binary interval consensus," in Proc. IEEE International Conference on Computer Communications (INFOCOM), San Diego, CA, Mar. 2010, pp. 1 9.

[5] S. Shang, P. Cuff, P. Hui, and S. Kulkarni, "An upper bound on the convergence time for quantized consensus," in Proc. IEEE International Conference on Computer Communications (INFOCOM), Turin, Italy, Apr. 2013, pp. $600-604$.

[6] A. Abdaoui, T. Elfouly, and M. Draief, "Distributed binary consensus algorithm and wireless sensor network," in Proc. 9th International Wireless Communications and Mobile Computing Conference (IWCMC), Sardinia, Italy, Jul. 2013, pp. 1331 - 1336.

[7] N. Al-Nakhala, R. Riley, and T. Elfouly, "Binary consensus in sensor motes," in Proc. 9th International Wireless Communications and Mobile Computing Conference (IWCMC), Sardinia, Italy, Jul. 2013, pp. 1337 - 1342.

[8] L. Zheng, Y. Yao, M. Deng, and S.-T. Yau, "Decentralized detection in ad hoc sensor networks with low data rate inter sensor communication," IEEE Transactions on Information Theory, vol. 58, no. 5, pp. 32153224, May 2012.

[9] Y. Mostofi, "Binary consensus with gaussian communication noise: A probabilistic approach," in Proc. 46th IEEE Conference on Decision and Control (CDC), New Orleans, LA, Dec. 2007, pp. 2528 - 2533.

[10] M. MalmirChegini, Y. Ruan, and Y. Mostofi, "Binary consensus over fading channels: A best affine estimation approach," in Proc. IEEE Global Telecommunications Conference (GLOBECOM), New Orleans, LO, Dec. 2008.

[11] Y. Ruan and Y. Mostofi, "Binary consensus with soft information processing in cooperative networks," in Proc. 47th IEEE Conference on Decision and Control (CDC), Cancun, Mexico, Dec. 2008, pp. 36133619 .

[12] Y. Mostofi and Y. Yuan, "Impact of heterogeneous link qualities and network connectivity on binary consensus," in Proc. American Control Conference (ACC), St. Louis, MO, Jun. 2009, pp. 1821-1826.

[13] Y. Mostofi and M. MalmirChegini, "Binary consensus over fading channels," IEEE Transactions on Signal Processing, vol. 58, no. 12, pp. 6340-6354, Dec. 2010.

[14] S. Ashrafi, M. MalmirChegini, and Y. Mostofi, "Binary consensus for cooperative spectrum sensing in cognitive radio networks," in Proc. IEEE Global Telecommunications Conference (GLOBECOM), Houston, TX, Dec. 2011.

[15] Y. Wang and P. Djuric, "Reaching consensus on a binary state by exchanging binary actions," in Proc. IEEE International Conference on Acoustics, Speech and Signal Processing (ICASSP), Kyoto, Japan, Mar. 2012, pp. $3297-3300$.

[16] — "A gossip method for optimal consensus on a binary state from binary actions," IEEE Journal of Selected Topics in Signal Processing, vol. 7, no. 2, pp. 274 - 283, Apr. 2013.

[17] R. Olfati-Saber and R. Murray, "Consensus problems in networks of agents with switching topology and time-delays," IEEE Transactions on Automatic Control, vol. 49, no. 9, pp. 1520 - 1533, Sep. 2004.

[18] R. Olfati-Saber, J. Fax, and R. Murray, "Consensus and cooperation in networked multi-agent systems," Proceedings of the IEEE, vol. 95, no. 1 , pp. 215 - 233, Jan. 2007.

[19] R. Saber and R. Murray, "Consensus protocols for networks of dynamic agents," in Proc. American Control Conference (ACC), Denver, CO, Jun. 2003, pp. $951-956$.

[20] R. Olfati-Saber, "Distributed Kalman filter with embedded consensus filters," in Proc. 44th IEEE Conference on Decision and Control and European Control Conference (CDC-ECC), Seville, Spain, Dec. 2005, pp. $8179-8184$.

[21] R. Olfati-Saber and J. Shamma, "Consensus filters for sensor networks and distributed sensor fusion," in Proc. 44th IEEE Conference on Decision and Control and European Control Conference (CDC-ECC), Seville, Spain, Dec. 2005, pp. 6698 - 6703.

[22] S. Boyd, L. E. Ghaoui, E. Feron, and V. Balakrishnan, Linear Matrix Inequalities in System and Control Theory, ser. Studies in Applied Mathematics. Philadelphia, PA: SIAM, 1997, vol. 15.

[23] P. Gahinet, A. Nemirovski, A. J. Laub, and M. Chilali, "LMI Control Toolbox," The MathWorks, Inc., Natick, MA, Tech. Rep., 1995. 